\title{
Mechanisms of thrombophilia
}

Editorial

Autor: Pablo García de Frutos

The term thrombophilia describes the predisposition to venous thromboembolism and, under certain circumstances, also an increased risk of arterial thrombosis. In a broad sense, thrombophilia includes any inherited or acquired disorder associated with an increased tendency to thrombosis. It was soon realised that thrombotic events have a complex multifactorial cause, usually a combination of genetic factors combined with acquired haematological disorders. Besides, behavioural factors, as diet or exercise, influence the incidence of thrombosis, making it difficult to asses the risk of thrombosis in a given individual. In any case, inherited thrombophilia is one of the main determinants of venous thromboembolism (VTE), and the presence of inherited thrombophilic defects exposes carriers to increased risks for VTE compared with non-carriers. The presence of hereditary thrombophilia should be considered in patients with a documented unexplained thrombotic episode or a positive family history. There is no clear relationship between clinical manifestations and the type of underlying thrombophilic defect. Thus, diagnosis of inherited thrombophilia has to be established on a laboratory basis.

The concept that thrombophilia could be associated with a genetically determined hypercoagulable state was first proposed already in 1965 after the discovery of an association of thrombosis and antithrombin deficiency in a large family. Further

studies in this field have gone in parallel with a better understanding of the components of blood coagulation, and the natural anticoagulant mechanisms essential in haemostasis, in particular the activated protein C pathway. In turn, these studies have lead to better therapies and protocols for the prophylactic control of thrombosis. Since the seminal work on antithrombin, genetic studies on thrombophilic families in combination with epidemiological studies lead to the discovery of anticoagulant protein deficits linked to thrombosis: protein $\mathrm{C}$, protein $\mathrm{S}$ and antithrombin. These deficiencies are generally caused by a series of deleterious mutations in the respective genes behaving as relatively rare autosomal dominant genetic traits with a low or incomplete penetrance. Still, very few cases of thrombosis could be explained by these mechanisms.

This situation changed with the discovery of activated protein C (APC) resistance in 1993 and the mutation associated with factor $\mathrm{V}$ Leiden (FV)Leiden, and, shortly afterwards, the discovery of a mutation in the 3' untranslated region of the prothrombin gene associated with increased concentration of prothrombin in plasma. Large epidemiological studies have confirmed that these mutations are risk factors for thrombophilia and opened the search for other thrombophilic traits. During the last decade, several additional prothrombotic risk factors have been described, including genetic variants and haplotypes associated with qualitative or quantitative defects of coagulation factor inhibitors, increased levels or function of coagulation factors, defects of the fibrinolytic system, altered platelet function, and hyperhomocysteinemia. At present, it is possible to identify an inherited predisposition to thrombosis in patients with such complications in about $50 \%$ to $80 \%$ of cases, depending on the study population. Nevertheless, despite the great number of mutations or polymorphisms which have been added to the list of candidate genetic traits implicated in thrombosis, the diagnosis of thrombophilia in most settings is still based on the "classical" parameters mentioned. In general, many of the genetic variants or haplotypes that have been recently studied are relatively common in the general population. These variants have been shown sometimes to be functional, i.e. they have an effect on the activity of the gene, but usually have only a minor impact on the thrombotic risk. 
Most of them are not interesting for diagnostic purposes, as they do not add significantly to the risk of thrombosis. In any case, genetic studies have provided insights into specific mechanisms of the coagulation cascade, which lead to thrombus formation as opposed to mechanisms of physiological haemostasis.

The present set of articles in this Theme Issue are devoted to novel aspects of the mechanisms linked to thrombophilia, and nicely illustrate how the combination of biochemical and genetic studies have provided a substantial advance in our field. The first three articles discuss and summarise our knowledge about a candidate gene, each one found to be implicated in the risk of thrombosis. Mutations in the genes coding for factor $V$ (F5) and protein S (PROS1) are well established as risk factors for thrombosis. Activated factor $\mathrm{V}$ is a cofactor of the prothrombinase complex essential in the exponential phase of thrombin production, but the discovery of factor $\mathrm{V}(\mathrm{FV})$ Leiden lead to the proposal of a new anticoagulant role for FV. Mutations in FV lead to an altered haemostatic balance, but manifest either as thrombotic or bleeding events. The article by Segers et al. describes how these functions are regulated and its implication in the aetiology of thromboembolic disease. As mentioned by the authors, FV variants are often found in combination with other prothrombotic factors in the same individual. Gene-gene interactions have been observed among the two alleles in F5, and mutations in the F5 gene as the FVLeiden could be combined with F5 haplotypes with a minor effect, as the F5 R2, to increase the risk of thrombosis. Besides these genetic interactions, acquired thrombotic traits could affect specifically FV, such as anti-FV auto-antibodies.

A second chapter in this series focuses on PROS1, the gene coding for the vitamin Kdependent anticoagulant protein S. Protein $S$ deficiency has always been the most difficult to study among prothrombotic deficiencies, due to intrinsic difficulties

in its diagnosis. Difficulties derive in part from the presence in plasma of two protein $S$ pools, either as a free form, or in complex with C4 binding protein. Additionally, protein S has no direct enzymatic activity, acting as a modulator of serine proteases, either activating APC or directly inhibiting the prothrombinase complex, making it difficult to standardize an activity assay in plasma and to establish normal ranges for protein $S$ assays in the general population. Despite these limitations, recent studies have raised the number of mutations associated with protein S deficiency (PSD) up to almost 200. Especially important have been the finding of large deletions in PROS1 in families were the initial genetic analysis was unable to find a causative mutation. Besides, the careful analysis of the mechanism associated with PSD has provided a better understanding of the relationship between the different kinds of PSD and the associated risk of thrombosis. New evidence support the concept that qualitative or mild quantitative deficiencies of protein $S$ could play a role in thrombophilia, which could be important in certain populations. While these two articles focus on new aspects of the classical mechanisms of inherited thrombophilia, the following article in the series by Hernández-Espinosa et al. focuses on the serpin family of protease inhibitors and proposes new mechanisms leading to thrombosis that go beyond the genetic inheritance of

deficiency. The atypical structure of serpins, the main direct inhibitors of thrombin and other serine proteases in plasma, makes this family of molecules especially sensitive to changes in environmental parameters such as $\mathrm{pH}$ or temperature. The concomitant effect of environmental and structurally de-stabilizing allelic variants could be the underlying cause of thrombosis associated with microbial infections. Furthermore, the described effect of certain drugs could also cause deficiency of haemostatic serpins that may be explained by conformational mechanisms. 
Interestingly, one of the latest components added to the natural anticoagulant pathways, the protein Z-dependent protease inhibitor (SERPINA10), belongs to the serpin family and it is actively studied as a candidate gene in thrombophilia. The article by Medina et al. reviews the interesting addition of a potentially important new candidate gene in the field of thrombosis. The discovery of a second protein C-binding protein in the endothelial cell membrane after thrombomodulin in 1995 added a new level of complexity to the APC anticoagulant system. The endothelial protein $C$ receptor (EPCR) has been shown to have a major impact both in the activation and in the anticoagulant activity of APC. During recent years, a wealth of genetic data on the EPCR gene has supported its important role in maintaining haemostasis. From these studies, some of the polymorphisms and haplotypes found in the EPCR gene emerge as possible factors affecting the risk of thrombosis. The haplotypes in EPCR clearly correlate with the soluble concentration of EPCR in plasma.

The last article in the present Theme Issue series studies a different aspect of the relationship between genetic variants and thrombosis, in this case not as a causative mechanisms underlying thrombosis. The article by Oldenburg et al. focuses on the effect of common genetic variants in the efficacy of the oral anticoagulant therapy. For some years it has been known that the oral anticoagulants could have broad differences in their effect. While some of the differences are clearly related to diet and other behavioural factors, there was also a genetic component in these differences, as was proved when genetic variants in the cytochrome P450 CYP2C9 were shown to affect the efficacy of oral anticoagulants. The discovery of the target enzyme for oral anticoagulants, VKORC1, and genetic studies determining the effect of different haplotypes of the gene with the efficacy of oral anticoagulants has lead to the conclusion that VKORC1 is the most important determinant of efficacy of the anticoagulant treatment. This discovery was important for establishing better dosing schemes for patients on anticoagulation, and therefore has had a direct consequence in clinical practice.

All the articles in the series are good examples of how genetic studies have shown the way for a better understanding of thrombosis and how to treat it. Still, it is a matter of intense debate under which circumstances genetic screening for thrombophilia are useful in clinical practice, as it seems advisable that it should be performed only in cases where such testing is likely to influence patient management, as it is clearly illustrated in the last article of the series. Furthermore, the knowledge obtained from the association of genetics and thrombotic disease has been central in establishing our present paradigm of haemostasis and its associated diseases. 